\title{
Rückfallbegünstigende und rückfallprotektive Faktoren nach der Entlassung aus dem Maßregelvollzug
}

\author{
Peggy Walde ${ }^{1}$ Vivien Lungwitz ${ }^{2}$ \\ Eingegangen: 10. Juni 2020 / Angenommen: 12. Juni 2020 / Online publiziert: 10. Juli 2020 \\ (c) Der/die Autor(en) 2020
}

\section{Zusammenfassung}

Im vorliegenden systematischen Review werden Faktoren zusammengetragen, welche den Erfolg der Legalbewährung bei entlassenen Patienten des Maßregelvollzugs ( $\$ \S 63$ und 64 StGB) positiv oder negativ beeinflussen können. Als Ergebnis einer Literaturrecherche konnten 7 häufig untersuchte Bereiche identifiziert werden: Wohnumgebung, Erwerbstätigkeit, Familie/Partnerschaft, Freizeit/soziale Kontakte, Substanzkonsum, Nachsorge und psychologische Variablen. Aus den Ergebnissen wurden, wo möglich, Daten zur Quantifizierung von Größe und Richtung der Effekte berechnet. Diese zeigen für Patienten des psychiatrischen Maßregelvollzugs ein erhöhtes Rückfallrisiko bei Substanzmissbrauch und häufigem Wechsel von Wohnort und Arbeitsplatz. Protektiv wirken dagegen ein psychisch stabiler Zustand und ein zumindest während der ersten Zeit nach der Entlassung institutionalisiertes Wohnumfeld. Für Patienten, die nach $\S 64$ StGB untergebracht sind, können keine eindeutigen Aussagen getroffen werden. Auch hier scheint jedoch erneuter Suchtmittelkonsum das Risiko für Rückfälle zu erhöhen.

Schlüsselwörter Systematischer Review · Forensische Psychiatrie · Psychisch kranke Straftäter · Deliktrückfälligkeit · Delinquenz

\section{Risk and protective factors associated with recidivism following release from forensic treatment}

\begin{abstract}
This systematic review gives a summary of factors which can positively or negatively affect the success of legal probation of patients released from forensic commitment (§ 63 and $\S 64$ StGB, German penal code). A systematic literature search identified seven frequently investigated domains: living environment, employment, family/partnership, leisure time/social associates, substance abuse, (forensic) psychiatric aftercare, and psychological variables. Whenever possible, summary data were calculated from the results to quantify the size and direction of effects. For patients from forensic psychiatric commitment, substance abuse and numerous changes in accommodation and employment correlated with an increased risk for recidivism. In contrast, a stable psychiatric condition and an institutionalized accommodation (at least for the time after transition into freedom) were identified to be protective variables. For patients committed under $\$ 64$ StGB (substance addiction) no clear statements can be made; however, it seems that renewed substance abuse after release increases the risk of recidivism.
\end{abstract}

Keywords Systematic review $\cdot$ Forensic Mental Health $\cdot$ Mentally ill offender $\cdot$ Reoffending $\cdot$ Addicted Offender

Zusatzmaterial online Zusätzliche Informationen sind in der Online-Version dieses Artikels (https://doi.org/10.1007/s11757020-00615-y) enthalten.

Peggy Walde, M.Sc.

peggy.walde@med.uni-rostock.de
1 Klinik für Forensische Psychiatrie, Universitätsmedizin Rostock, Gehlsheimer Str. 20, 18147 Rostock, Deutschland

2 Institut für Psychologie, Technische Universität Chemnitz, Wilhelm-Raabe-Str. 43, 09120 Chemnitz, Deutschland 


\section{Einleitung}

Im Rahmen der Behandlung psychisch kranker und suchtkranker Straftäter stellt sich u. a. die Frage, wie ehemalige Patienten des Maßregelvollzugs erfolgreich in die Gesellschaft reintegriert werden können und wo die größten Gefahren für den Erfolg der Legalbewährung liegen. Zusätzlich spielen protektive Faktoren in der Behandlung eine zunehmende Rolle. Die Wirksamkeit von Kriminaltherapie konnte bereits metaanalytisch belegt werden (Andrews et al. 1990). Auch Faktoren, welche kriminelles Verhalten vorhersagen, gelten als allgemein gut belegt (Andrews und Bonta 2010). Die Studienlage wird dünner, wenn es um psychisch kranke Straftäter und deren Rückfälligkeit in strafrechtlich relevantes Verhalten geht. Eine Metaanalyse kommt zu der Einschätzung, dass soziodemografische Variablen, kriminelle Vorgeschichte, devianter Lebensstil sowie klinische Variablen eine Rolle spielen (Bonta et al. 1998). Allerdings handelt es sich bei den untersuchten Einflussfaktoren überwiegend um unveränderliche Faktoren. Sie bieten damit keine verwertbaren Informationen für die praktische Arbeit nach der Entlassung. Studien aus dem deutschsprachigen Raum, welche dynamische Faktoren nach erfolgter Kriminaltherapie bei Strafgefangenen berücksichtigen, kommen u. a. von Dahle (2005). Es zeigte sich, dass u. a. Art und Umfang der sozialen Einbindung, eine intakte Partnerschaft und Arbeit nach der Entlassung mit geringeren Rückfallraten assoziiert waren. Die Übertragbarkeit auf eine Population des Maßregelvollzugs ist aufgrund der postulierten Ähnlichkeit zwischen Strafgefangenen und Maßregelpatienten bedingt möglich, aber nicht völlig kritiklos. Parallelen zwischen den Gruppen zeigen sich insbesondere hinsichtlich der familiären und delinquenten Vorbelastung (Leygraf 1988). Des Weiteren weisen auch viele Insassen des Regelvollzugs ein breites Spektrum psychischer Störungen auf. Art und Verteilung der Störungen unterscheiden sich aber von denen des Maßregelvollzugs (z. B. Dudeck et al. 2009; von Schönfeld et al. 2006). Zudem werden bei Maßregelpatienten diese Störungen als grundlegend für deren delinquentes Verhalten angesehen. Der Gesetzgeber geht in ihrem Fall davon aus, dass eine Behandlung der psychischen Störung auch die Gefahr weiterer Straftaten deutlich verringert oder sogar beseitigt. Insbesondere Patienten des Maßregelvollzugs nach $\S 63$ StGB erhalten eine oft jahrelange Therapie mit zahlreichen Lockerungsschritten und Erprobungen, bevor sie bedingt entlassen werden. Seit der Reform des Gesetzes zur Führungsaufsicht im Jahr 2007 kann zusätzlich eine ambulante Nachbetreuung zur Auflage gemacht werden. Diese Möglichkeiten - Therapie und Nachbehandlung - steht den meisten Insassen des Regelvollzugs nicht zur Verfügung. Des Weiteren ergeben sich aus der psychischen Erkrankung zusätzliche Faktoren, die für ein Rückfallgeschehen relevant sein können (z.B.
Medikamenten-Compliance oder spezifische Schwierigkeiten in zwischenmenschlichen Beziehungen aufgrund einer doppelten Stigmatisierung als psychisch krank und kriminell). Hinzu kommen methodische Aspekte, welche eine separate Betrachtung rückfallbegünstigender und -protektiver Faktoren für Maßregelpatienten sinnvoll erscheinen lassen. Beispielsweise führt Schalast (2019) aus, dass einer Unterbringung im Maßregelvollzug eine erhebliche Deliktschwere zugrunde liegt. Ein unreflektierter Vergleich einer Gefängnis- und einer Maßregelstichprobe würde demzufolge zu ungleichen Ausgangsbedingungen und zur Konfundierung populationsspezifischer Störvariablen führen.

\section{Methode}

Zur Identifikation geeigneter Studien wurde eine elektronische Datenbankrecherche durchgeführt. Diese fand im Zeitraum von April bis Mai 2018 statt und umfasste die folgenden Datenbanken: The Cochrane Library, MEDLINE, PsycARTICLES, PsycINFO, PSYNDEX, PubMed, Scopus, KrimDok und KrimLit. Zusätzlich wurden eine Vorwärtsund eine Rückwärtssuche anhand passender Artikel durchgeführt sowie die Tagungsbände relevanter Fachveranstaltungen und die Inhaltsverzeichnisse verschiedener Fachzeitschriften durchgesehen. Zur Identifikation laufender Studien wurde das Trial-Register clinicaltrials.gov durchsucht. Es wurde außerdem versucht, Ergebnisse aus unveröffentlichten Studien durch direkte Korrespondenz mit den entsprechenden Autoren zu finden.

In den Review eingeschlossen wurden Studien, welche entlassene Patienten des Maßregelvollzugs nach $\S 63$ oder $\S 64$ StGB untersuchten, unabhängig von der Grundlage ihrer Entlassung (regulär, Abbruch etc.). Des Weiteren sollte mindestens ein Outcome zur Deliktrückfälligkeit enthalten sein sowie mindestens eine dynamische Variable, deren Einfluss mit dem Deliktrückfall in Zusammenhang gebracht wurde. Wichtig dabei war, dass die dynamische Variable nach der Entlassung erhoben wurde, nicht davor (z.B. im Rahmen von Entlassungsgutachten), da eben der Einfluss dieser sich erst nach der Entlassung ergebenden Faktoren, Gegenstand der vorliegenden Untersuchung war. Einzelfalluntersuchungen wurden ausgeschlossen. Weiter wurden hinsichtlich des Studiendesigns keine Einschränkungen gemacht. Ebenso wurden keine Einschränkungen hinsichtlich des Publikationszeitraumes und der Sprache bei der Datenbankrecherche gemacht. Die Sprache der ausgewerteten Publikationen beschränkte sich jedoch auf Deutsch und Englisch.

Die eingeschlossenen Primärstudien wurden auf mögliche Verzerrungen geprüft. Aufgrund der heterogenen Studiendesigns wurden verschiedene Tools zur Bewertung herangezogen und an die eigenen Bedürfnisse angepasst (ACRO- 
BAT-NRSI, Sterne et al. 2014; Checkliste von Moga et al. 2012).

Um Unterschiede zwischen verschiedenen Gruppen feststellen zu können, wurden $\mathrm{X}^{2}$-Tests berechnet, sofern diese nicht bereits in den Studien berechnet waren. Um die Größe des Effekts einzuschätzen und Studien vergleichbar zu machen, wurde zusätzlich Cohens Omega $(\omega)$ berechnet. Die Interpretation orientiert sich an den Konventionen nach Cohen (Cohen 1988), 0,1 = kleiner Effekt, 0,3 = mittlerer Effekt, $0,5=$ großer Effekt.

\section{Ergebnisse}

\section{Literaturrecherche}

Insgesamt wurden 25 relevante Publikationen identifiziert, die auf 16 Primärstudien zurückgingen. Eine Studie mit 53 Patienten (Bischoff-Mews 1989) wurde im Nachhinein ausgeschlossen, da anhand der publizierten Angaben keine Daten zuverlässig entnommen werden konnten. Die verbliebenen 15 Primärstudien sind in Tab. 1 dargestellt.

Die Deliktrückfälligkeit wurde in 8 Studien anhand der Bundeszentralregisterauszüge (BZR) erhoben; sechs weitere Untersuchungen stützten sich auf Unterlagen der Bewährungshilfe, Führungsaufsichtsbehörde bzw. forensischer Ambulanzen. In einer Publikation war die Informati-

Tab. 1 Eingeschlossene Primärstudien

\begin{tabular}{|c|c|c|c|c|}
\hline AutorIn & Jahr & $\begin{array}{l}\text { Rec } \\
\text { lage } \\
\text { StG }\end{array}$ & $n\left(\right.$ davon $\left.\sigma^{7}\right)$ & Untersuchte Risiko-/protektive Faktoren \\
\hline Bezzel & 2013 & 64 & 127 (n. a.) & $\mathrm{S}$ \\
\hline Butz & 2016 & $\begin{array}{l}63 \\
64\end{array}$ & $\begin{array}{l}155(143) \\
251(231)\end{array}$ & $\begin{array}{l}\text { W, A, FS, FP, S, Kr; beobachtete kritische Verhaltensweisen, realistische } \\
\text { Zukunftspläne, von Therapeuten zugesprochene gute Chancen auf soziale und } \\
\text { berufliche Reintegration laut Therapeut }\end{array}$ \\
\hline Dimmek & 2012 & 63 & $246(234)$ & W, A, FS, S, Wei; kritische Lebensereignisse \\
\hline $\begin{array}{l}\text { Dimmek } \\
\text { et al. }\end{array}$ & 2010 & 64 & $136(130)$ & A, F, S, realistische Lebensvorstellungen \\
\hline Gretenkord & 2001 & 63 & $247(188)$ & $\mathrm{W}, \mathrm{A}, \mathrm{FS}, \mathrm{S}$ \\
\hline Hahn & 2007 & $\begin{array}{l}63 \\
64\end{array}$ & $\begin{array}{l}10(10) \\
2(2)\end{array}$ & $\begin{array}{l}\text { FS, FP, F, NA, Gefühle von Verstehbarkeit, Sinnhaftigkeit, Handhabbarkeit, } \\
\text { Adaptation relevanter Therapieergebnisse in echte Lebenszusammenhänge, Hoffnung/ } \\
\text { Optimismus/Lebenszufriedenheit } \\
\text { Besserung deliktfördernder psychiatrischer Symptomatik, Empathiefähigkeit, } \\
\text { Fähigkeit zu Erkennen und Einhalten eigener Grenzen und Konsequenzen eigener } \\
\text { Handlungen/Introspektionsfähigkeit und Fähigkeit zur Herstellung sinnhafter Bezüge } \\
\text { zwischen eigenem Verhalten und eigener Lebensperspektive, soziale Flexibilität/ } \\
\text { Anpassungsfähigkeit, Fähigkeit zur Distanzierung von neg. Einflüssen, } \\
\text { Selbstwirksamkeitserleben, Verfügbarkeit und Akzeptanz von externen/ } \\
\text { institutionalisierten Kontrollinstanzen, deren bedarfsorientierte Ausgestaltung, } \\
\text { Balance externer und interner Protektivfaktoren }\end{array}$ \\
\hline Hartl & 2012 & $\begin{array}{l}63 \\
64\end{array}$ & $\begin{array}{l}128(127) \\
994(912)\end{array}$ & W, A, FS, FP, F, Wei, Kr, rechtliche Betreuung \\
\hline Koch & 1988 & 64 & $102(88)$ & W, A, F, S, NA, Selbsthilfegruppe, Kinder \\
\hline $\begin{array}{l}\text { Müller- } \\
\text { Isberner }\end{array}$ & 1996 & 63 & 123 (n. a.) & NA \\
\hline $\begin{array}{l}\text { Passow } \\
\text { et al. }\end{array}$ & 2015 & 64 & $41(36)$ & $\mathrm{S}, \mathrm{NA}$ \\
\hline Pfaff & 1998 & 64 & 77 (n. a.) & A, FP, S, NA \\
\hline Ritzel & 1978 & $63^{\mathrm{a}}$ & $501(457)$ & W, A, FS, FP, F, S, Kr, NA \\
\hline $\begin{array}{l}\text { Schmidt- } \\
\text { Quernheim }\end{array}$ & 2011 & 63 & $44^{\text {b }}$ (n. a.) & W, A, FS, FP, F, S, Kr \\
\hline Seifert & 2010 & 63 & 202 (n. a.) & W, A, FS, FP, NA \\
\hline $\begin{array}{l}\text { Seifert } \\
\text { et al. }\end{array}$ & 2018 & 63 & $333(303)$ & W, A, FS, FP, NA \\
\hline
\end{tabular}

$n$ Anzahl der Probanden, Rechtsgrundlage $§ 63 / 64$ StGB, n. a. nicht angegeben, $S$ Substanzkonsum, $W$ Wohnen, $A$ Arbeit oder vergleichbare Beschäftigung, FS Freizeit und Sozialkontakte, FP Familie und Partnerschaft, Kr Krisen, F Finanzen, Wei Weisungsverstöße, NA Nachsorge aBzw. § 42b StGB vor 1975

${ }^{\mathrm{b}}$ Teilstichprobe der gematchten Patienten 
onsquelle nicht ersichtlich; eine weitere Studie untersuchte explizit protektive Faktoren bei rückfallfreien Teilnehmern. In 14 Publikationen erfolgte die Betrachtung retrospektiv; zwei Studien waren prospektiv angelegt.

Die Erhebung dynamischer Faktoren erfolgte in der Regel über Akteneinsicht. Grundlage bildeten in 9 Studien Berichte von Bewährungshelfern, Verlaufsdokumentationen forensischer Ambulanzen oder Unterlagen der entlassenden Klinik. Sechs Studien befragten die teilnehmenden Patienten bzw. Bewährungshelfer direkt. Aus einer Studie geht auch hier keine Informationsquelle hervor.

In allen Studien zusammen wurden 2714 Patienten des Maßregelvollzugs untersucht, davon waren 1685 Patienten nach $\S 63$ StGB untergebracht und 1029 Patienten nach $\S 64$ StGB. Alle Studien, die Patienten nach $\S 64$ StGB betrachteten, beschränkten sich auf regulär entlassene Patienten. Es lagen keine Studien zum Bewährungsverlauf von Therapieabbrechern vor.

Da die untersuchten Patienten nicht alle zur selben Zeit entlassen waren, wurden in den Studien durchschnittliche Katamnesezeiträume beschrieben. Diese betrugen für alle Studien mindestens 12 Monate, in 4 Studien durchschnittlich unter 3 Jahre, in 8 Studien zwischen 3 und 5 Jahre und in 3 Studien über 5 Jahre. Eine Publikation nannte keine Durchschnittswerte.

In 8 Studien stammten alle untersuchten Patienten aus einer Einrichtung. Fünf Studien waren multizentrisch angelegt, beschränkten sich aber auf ein Bundesland. Zwei Untersuchungen bezogen sich auf eine bundesweite Erhebung; in einer Publikation waren die regionalen Gegebenheiten nicht näher benannt.

\section{Definition und Erfassung der Outcomes}

Die Definition der Deliktrückfälligkeit wurde in den gefundenen Studien sehr heterogen gehandhabt. Untersuchungen, die sich auf Auszüge des Bundeszentralregisters (BZR) stützten, werteten in der Regel jeden neuen Eintrag als Rückfall (erneute Straftaten ebenso wie Verstöße gegen richterliche Weisungen). Einige Autoren differenzierten die Einträge weiter aus, z.B. nach Deliktschwere (z.B. Dimmek 2012; Seifert 2010) oder Deliktgruppe (z. B. Gretenkord 2001; Müller-Isberner 1996). Ähnliche Ausdifferenzierungen finden sich auch in Studien, die sich auf andere Quellen stützten.

Nach Sichtung aller Studien bot sich die Einteilung der Ergebnisse in folgende 7 Kategorien an: Wohnen, Arbeit, Familie und Partnerschaft, soziale Kontakte und Freizeit, Substanzkonsum, Nachsorge und psychologische und störungsassoziierte Variablen.

Die große Varianz hinsichtlich Studiendesign, methodischer Qualität und Outcome-Darstellung machte eine übersichtliche Zusammenfassung schwierig. Im Folgenden wird daher schwerpunktmäßig auf Studien eingegangen, die bei der Bewertung des Verzerrungsrisikos vergleichsweise günstig abgeschnitten haben. Die vollständigen Ergebnisse sind im Zusatzmaterial online in den Tabellen S1-S7 aufgeführt. Bei den Berechnungen wurden Drop-outs berücksichtigt.

\section{Wohnen}

Die Wohnumgebung stellte einen der am häufigsten untersuchten Faktoren dar. Die verschiedenen Möglichkeiten der Unterkunft ließen sich für alle Patientengruppen grob in institutionalisierte und private Unterkünfte unterteilen.

Insbesondere für Patienten, die nach $\S 63$ StGB untergebracht waren, zeigte sich ein Einfluss der Erstunterkunft nach der Entlassung. Mehrere Studien berichteten von Unterschieden und (tendenziell) positiven Effekten bei Entlassung in ein institutionalisiertes Umfeld, z. B. Seifert (2010; $\left.\mathrm{X}^{2}=20,83, p<0,01, \omega=0,32\right)$. Insbesondere die Unterkunft in einem Übergangswohnheim erwies sich als vorteilhaft $(\mathrm{OR}=0,32,95 \%$-KI $[0,14,0,71], p<0,001)$. In eine ähnliche Richtung deuteten die Daten von Schmidt-Quernheim (2011; OR = 2,63 für Entlassung ins private Umfeld, 95\%KI $[0,63,11,00], p=0,187)$. Der Effekt schien allerdings im Verlauf der Bewährungszeit zu verschwinden. Der Einfluss der zuletzt erfassten Unterkunft nach einem Katamnesezeitraum von durchschnittlich 4,4 Jahren war praktisch nicht mehr vorhanden $(\mathrm{OR}=1,07,95 \%$-KI $[0,30,3,80]$, $p=0,920)$. Auch Gretenkord (2001) stellte Unterschiede hinsichtlich der Wohnform bei Entlassung fest. Patienten, die in institutionalisierte Einrichtungen entlassen wurden, wiesen seltener erneute Einträge im BZR und erneute Freiheitszentzüge auf. Auch Dimmek (2012) beschrieb den Einfluss der ersten Unterkunft nach der Entlassung im Hinblick auf erneute Verfahren $\left(\mathrm{X}^{2}=12,97, p=0,011, \omega=0,23\right)$. Auch hier war eine stärker strukturierte (institutionalisierte) Unterkunft tendenziell vorteilhafter. Die Effektstärke schwankte ein wenig in Abhängigkeit von der Primärdiagnose der Patienten. Die älteste Untersuchung zur Erstunterkunft nach der Entlassung stammte von Ritzel (1978). Nach Umstrukturierung der Daten aus der Originalpublikation konnten hier entsprechende Werte berechnet werden. Es konnte gezeigt werden, dass auch hier eine institutionelle Unterbringung bedeutsame Vorteile brachte $(\mathrm{OR}=0,41$, $95 \%$-KI [0,20, 0,85], $p=0,016)$. Der Einfluss der Erstunterkunft lag im geringen bis moderaten Bereich.

Des Weiteren erwies sich Beständigkeit als günstiger Faktor. Ein häufiger Wechsel der Unterkunft schien ein Hinweis auf dahinterliegende Probleme zu sein. Die Effektstärke bewegte sich im moderaten bis großen Bereich. Interessanterweise schien ein Wechsel der Unterkunft immer ungünstig zu sein, egal, aus welchem Grund er erfolgte. Selbst Patienten, die z. B. aufgrund einer neuen Arbeit um- 
zogen, hatten ein erhöhtes Risiko, erneut straffällig zu werden $(\mathrm{OR}=8,55,95 \%$-KI $[4,63,15,77], p<0,001)$. Denkbar wäre, dass ein Umzug stabilisierende soziale Strukturen zerstörte und der Patient möglicherweise mit zahlreichen neuen Unabwägbarkeiten überfordert war.

Für jene Patienten, die in das private Umfeld zurückkehrten, zeigten sich ebenfalls einige Tendenzen. Ungünstig erschien die Rückkehr in die Herkunftsfamilie. Dimmek (2012) beschrieb für die Gesamtstichprobe seiner Patienten ein nahezu doppelt so hohes Risiko erneuter Verfahren im Vergleich zu Patienten, die nach der Entlassung allein wohnten ( $\mathrm{OR}=1,90,95 \%$-KI $[0,62,5,81], p=0,258)$. Ritzel (1978) beschrieb ein etwa eineinhalbfach erhöhtes Risiko erneuter Straftaten $(\mathrm{OR}=1,54,95 \%$-KI $[0,80,2,96]$, $p=0,198)$. Durchwachsen erschien dagegen die Rückkehr zu einer eigenen Familie oder Partnerin. Seifert (2010) berichtete von einem potenziell protektiven Effekt gegenüber dem Alleinwohnen $(\mathrm{OR}=0,85,95 \%$-KI [0,31, 2,33], $p=0,750)$. Allerdings zeigte sich bei Patienten, welche mit einer Gewaltstraftat rückfällig wurden, bei Seifert et al. (2018) ein entgegengesetzter Effekt $(\mathrm{OR}=1,78$, 95\%-KI $[0,54,5,87], p=0,342)$. Nahezu keinen Unterschied zwischen Patienten, die bei ihren Partnern lebten, und jenen, die allein lebten, fand Dimmek (2012). Ritzel (1978) berichtete von einem deutlich positiven Effekt der Unterkunft bei der Partnerin im Vergleich zu Alleinwohnenden $(\mathrm{OR}=0,23$, $95 \%$-KI $[0,08,0,70], p=0,010)$.

Hinsichtlich der Wohnverhältnisse der Patienten, die nach $\S 64$ StGB untergebracht waren, gestaltete sich die Studienlage etwas übersichtlicher. Hier war es in keiner Studie möglich, eigene Werte zu berechnen. Butz (2016) und Hartl (2012) berichteten über die Wohnsituation bei Entlassung. Butz berichtete über keinerlei signifikante Unterschiede hinsichtlich des Einflusses der Entlassungsumgebung auf die erneute Straffälligkeit. Eine sehr geringe Rückfallquote erschwerte jedoch sowohl ihm als auch Hartl (2012) statistische Berechnungen. Letzterer verzichtete daher auf eine inferenzstatistische Auswertung seiner Daten und beschrieb diese lediglich deskriptiv. Patienten, die allein lebten, hatten mit einer Rückfallquote von 19,4\% etwas mehr Straftaten begangen als Patienten, die mit Partnern oder Familie lebten $(12,7 \%)$.

\section{Erwerbstätigkeit}

Für ehemalige Patienten nach $\S 63$ StGB stellte Seifert (2010) Unterschiede hinsichtlich der Legalbewährung fest. Eigene Berechnungen auf der Grundlage seiner Daten ergaben eine mittlere Effektstärke nach durchschnittlich 7,5 Jahren $(\omega=0,32)$. Ähnliche Ergebnisse zeigten sich bei derselben Stichprobe auch noch nach einem deutlich längeren Katamnesezeitraum ( $\omega=0,34$ nach 16,5 Jahren; Seifert et al. 2018). Die älteste Untersuchung hierzu stammte von
Ritzel (1978). Es zeigte sich ein Unterschied zwischen den einzelnen Kategorien zur Herkunft des Lebensunterhalts $\left(\mathrm{X}^{2}=14,10, p=0,001, \omega=0,18\right)$. Wer nach der Entlassung keine reguläre Tätigkeit aufnahm, hatte ein etwa 2,6-fach erhöhtes Risiko für Deliktrückfälligkeit $(\mathrm{OR}=2,59,95 \%$ $\mathrm{KI}[1,73,3,88], p<0,001, \omega=0,23)$. Eine geringere, statistisch nichtsignifikante Bedeutung hatte dagegen der Zeitpunkt der Arbeitsaufnahme $\left(\mathrm{X}^{2}=3,19, p=0,203, \omega=0,12\right)$. Eine negative Tendenz von Beschäftigungslosigkeit gegenüber einer Beschäftigung stellten Seifert et al. (2018) in Bezug auf gewalttätige Deliktrückfälle fest $(\mathrm{OR}=2,19,95 \%$ KI [0,94, 5,13], $p=0,071)$. Keinen signifikanten Einfluss der Art der Beschäftigung nach Entlassung und im katamnestischen Verlauf fand Butz (2016). Weiteren Fragen zum Arbeitsverhältnis ging Ritzel (1978) nach. Er untersuchte die Vorhersagekraft der Dauer des ersten Arbeitsverhältnisses, der Häufigkeit von Arbeitsplatzwechseln und von Schwierigkeiten am Arbeitsplatz. Die Höhe der Deliktrückfälligkeit unterschied sich nicht bedeutsam nach der Dauer des ersten Arbeitsverhältnisses (wenige Monate bis zu mehreren Jahre; $\left.\mathrm{X}^{2}=4,58, p=0,101, \omega=0,14\right)$. Wesentlich bedeutender war die Häufigkeit der Arbeitsplatzwechsel $\left(\mathrm{X}^{2}=30,80, p<0,001, \omega=0,38\right)$. Während ein bis zwei Arbeitsplatzwechsel (im Vergleich zu keinem Wechsel) im statistisch nichtbedeutsamen Bereich lagen, stieg der OR mit jedem weiteren Wechsel. Bei mehr als 5 Wechseln lag der OR bei 10,4 (95\%-KI [3,35, 32,26], $p<0,001)$. Auch die Angabe von Schwierigkeiten am Arbeitsplatz könnte ein Warnsignal sein $\left(\mathrm{X}^{2}=6,66, p=0,010, \omega=0,17\right)$. Teilnehmer, welche von Schwierigkeiten am Arbeitsplatz berichteten, wurden doppelt so häufig rückfällig wie Teilnehmer, die dies verneinten $(\mathrm{OR}=2,04,95 \%$-KI [1,18, 3,51], $p=0,001)$.

Über den Einfluss der Erwerbssituation nach der Entlassung ehemaliger \$-64-Patienten berichteten Dimmek et al. (2010). Für primär alkoholabhängige Patienten konnten Unterschiede hinsichtlich der Art des Beschäftigungsverhältnisses nur tendenziell gezeigt werden. Probanden in einem strukturierten Beschäftigungsverhältnis wurden etwas häufiger rückfällig als jene, die arbeitslos waren $(\mathrm{OR}=1,27$, $95 \%$-KI [0,43, 3,73], $p=0,711)$. Ein günstigerer Effekt zeigte sich bei den drogenabhängigen Patienten. Hier schien eine geregelte Beschäftigung einen protektiven Effekt $\mathrm{zu}$ haben $(\mathrm{OR}=0,26,95 \%$-KI $[0,07,0,98], p=0,047)$. Ebenfalls den Einfluss der Beschäftigung nach der Entlassung untersuchte Butz (2016). Er konnte keinen signifikanten Einfluss des ausgeübten Berufs zum Zeitpunkt der Aufnahme in die ambulante Nachsorge feststellen. Abseits von der Arbeitssituation bei Entlassung fragten Dimmek et al. (2010) die Mitarbeiter der entlassenden Klinik, wie diese die finanziellen Ressourcen der Patienten zum Zeitpunkt ihrer Entlassung einschätzten (eher ausreichend vs. eher nicht ausreichend). Für alkoholabhängige Patienten ergab 
sich kein nennenswerter Einfluss der eingeschätzten finanziellen Verhältnisse auf ihre Legalbewährung $\left(\mathrm{X}^{2}=0,19\right.$, $p=0,891, \omega=0,05)$. Für drogenabhängige Patienten ergab sich ein Unterschied zugunsten ausreichender finanzieller Ressourcen $\left(\mathrm{X}^{2}=4,06, p<0,05\right)$.

\section{Familie und Partnerschaft}

Für Patienten, welche nach $\S 63$ StGB untergebracht waren, ist anzumerken, dass eine Untersuchung dieses Faktors schwierig ist. Etwa drei Viertel der Patienten waren ledig. Das Zusammentreffen von Partnerschaft und Rückfall (für Maßregelpatienten des psychiatrischen Maßregelvollzugs ebenfalls vergleichsweise selten) ist daher eine seltene Kombination. Dennoch erhob eine Studie Daten für durchschnittliche Katamnesezeiträume von 7,5 Jahren (Seifert 2010) und 16,5 Jahren (Seifert et al. 2018). Es zeigte sich, dass sich das Bestehen einer Partnerschaft tendenziell ungünstig auswirkte (nach 7,5 Jahren: $O R=1,72,95 \%$ KI $[0,96,3,09], p=0,069)$. Noch ungünstiger fiel der Effekt für die Subgruppe jener aus, die in einem Katamnesezeitraum von durchschnittlich 16,5 Jahren mit Gewaltstraftaten rückfällig wurden $(\mathrm{OR}=2,39,95 \%$-KI $[1,14,5,03]$, $p=0,022)$. Einschränkend muss erwähnt werden, dass der Stand der Partnerschaft aus den Akten der Bewährungshelfer erhoben wurde, welche nur für den Zeitraum der Führungsaufsicht Berichte anfertigten. Da die Führungsaufsicht maximal 5 Jahre dauert, ist unklar, ob der in den Studien beschriebene Beziehungsstatus tatsächlich den aktuellen Stand nach 7,5 bzw. 16,5 Jahren widerspiegelt. Dieselbe Tendenz berichtete Schmidt-Quernheim (2011). Auch hier wirkte sich eine bestehende Partnerschaft ungünstig auf den Bewährungsverlauf aus (OR=1,90, 95\%-KI [0,56, 6,41], $p=0,300)$. Keinen signifikanten Einfluss des Beziehungsstatus innerhalb des 4-jährigen Katamnesezeitraums fand dagegen Butz (2016). Dies galt sowohl für den Beziehungsstatus zum Zeitpunkt der Aufnahme in die Nachsorgeambulanz als auch für die letzte Befragung 4 Jahre nach Entlassung.

Ähnlich durchwachsen fielen die Ergebnisse aus für Patienten, die nach $\S 64$ StGB untergebracht waren. Butz (2016) konnte keinen statistisch bedeutsamen Einfluss des Beziehungsstatus feststellen. Während Hartl (2012) einen eher ungünstigen Trend bei bestehenden Partnerschaften beschrieb, berichtete Pfaff (1998) eine protektive Wirkung. Beide Ergebnisse waren jedoch nicht signifikant. Hartl (2012) untersuchte zusätzlich den Einfluss von Elternschaft. Dieser schien bis zu einer bestimmten Anzahl im Haushalt lebender Kinder protektiv zu sein. Patienten, die mit einem Kind im Haushalt lebten, wurden deutlich seltener deliktrückfällig als jene, die keine Kinder hatten $(\mathrm{OR}=0,31,95 \%$-KI $[0,11,0,84], p=0,022)$. Die Rückfälligkeit über alle Kategorien (keine Kinder, 1, 2, 3,
$>3$ Kinder $)$ unterschied sich nicht nennenswert $\left(X^{2}=6,66\right.$, $p=0,155, \omega=0,16)$. Der Autor schlussfolgerte daraus, dass der protektive Effekt von Elternschaft ab einer bestimmten Anzahl von Kindern im Haushalt zu kippen schien.

\section{Freizeit und soziale Kontakte}

Für Patienten, die nach $\S 63$ StGB untergebracht waren, fand Schmidt-Quernheim (2011) Gruppenunterschiede hinsichtlich der Freizeitgestaltung $\left(\mathrm{X}^{2}=4,15, p=0,042\right.$, $\omega=0,30)$. Probanden, deren Freizeitgestaltung nach Einschätzung der behandelnden Therapeuten eher passiver oder problematischer Natur war, wurden über 3-mal häufiger mit erneuten Delikten auffällig als jene, die ihre Freizeit sinnvoll verbrachten $(\mathrm{OR}=3,71,95 \%$-KI [1,02, 13,47], $p=0,046)$. Diese Ergebnisse konnte Butz (2016) jedoch nicht bestätigen. Da Freizeitgestaltung auch der Pflege sozialer Kontakte dient, bietet sich die Frage nach der engsten Bezugsperson an. Eine ältere Untersuchung fand signifikante Unterschiede zwischen den Bezugspersonen $\left(\mathrm{X}^{2}=45,62, p<0,001, \omega=0,33\right.$; Ritzel 1978). Die Eltern als erste Bezugspersonen erwiesen sich als tendenziell günstiger im Vergleich zu keiner Bezugsperson $(\mathrm{OR}=0,63,95 \%$-KI $[0,25,1,62], p=0,046)$. Im Vergleich zu ihnen schnitten andere Personengruppen jedoch deutlich besser ab. Insbesondere sonstige Verwandte (hauptsächlich Geschwister oder erwachsene Kinder, OR=0,25, 95\%KI $[0,09,0,70], p=0,008)$, Freundin bzw. Ehepartnerin $(\mathrm{OR}=0,23,95 \%$-KI $[0,08,0,70], p=0,010)$ und das Personal institutioneller Einrichtungen $(\mathrm{OR}=0,12,95 \%$ KI [0,05, 0,33], $p<0,001)$ erzielten signifikante Effekte. Tendenziell ebenfalls günstiger war der Einfluss fremder Betreuungspersonen ( $\mathrm{OR}=0,42,95 \%$-KI $[0,16,1,11]$, $p=0,081)$. Inwiefern der Kontakt zur Herkunftsfamilie förderlich war, untersuchte Seifert (2010). Er fand keine Unterschiede zwischen Probanden mit und ohne Kontakt zur Familie. Die Tendenz war jedoch eher ungünstig, d.h., Probanden mit Kontakt zur Familie wurden häufiger rückfällig ( $\mathrm{OR}=1,26,95 \%$-KI $[0,96,3,09], p=0,069)$. Hinsichtlich gewalttätiger Rückfalldelikte erwies sich der Kontakt zur Familie sogar als äußerst ungünstig ( $O R=5,21$, $95 \%$-KI [1,21, 22,43], $p=0,027)$. Das bloße Vorhandensein einer Bezugsperson sagt jedoch mitunter nicht viel über die Qualität der Beziehung aus. Dieser Frage ging Ritzel (1978) nach. Er fand deutliche Unterschiede in den Rückfallraten in Abhängigkeit von der Beziehungsqualität $\left(\mathrm{X}^{2}=24,88, p<0,001, \omega=0,33\right)$. Anders, als man vermuten könnte, wurden Patienten, die ein ambivalentes bzw. (im Fall von Personal institutioneller Einrichtungen) professionelles Verhältnis zu ihrer engsten Bezugsperson hatten, seltener rückfällig als jene Patienten, die ein gutes bzw. sehr gutes Verhältnis zu ihrer Bezugsperson pflegten $(\mathrm{OR}=0,56$, $95 \%$-KI [0,37, 0,87], $p=0,01)$. Möglicherweise spielt hier 
eine Konfundierung professioneller und privater Bezugspersonen eine Rolle. Tendenziell anders herum verhielt es sich, wenn das Verhältnis zur engsten Bezugsperson schlecht oder sehr schlecht war. Diese Patienten wurden mehr als doppelt so häufig erneut straffällig wie jene mit einem guten Verhältnis zu ihren engsten Bezugspersonen $(\mathrm{OR}=2,47,95 \%$-KI $[0,13,4,58], p=0,004)$.

Für Patienten, die nach $\S 64$ StGB untergebracht waren, fand Butz (2016) keine bedeutsamen Zusammenhänge zwischen Freizeitaktivität und Legalbewährung $\left(\mathrm{X}^{2}=3,60\right.$, $p=$ n. s. [Angaben aus Publikation], $\omega=0,13$ ).

\section{Substanzkonsum}

Für Patienten, die nach $\S 63$ StGB untergebracht waren, stellte bereits Ritzel (1978) Zusammenhänge zwischen Alkoholkonsum nach der Entlassung und erneuter Straffälligkeit fest $\left(X^{2}=55,72, p<0,001 \omega=0,39\right)$. Probanden, die Alkohol im Übermaß konsumierten oder einen Missbrauch/ Suchtverhalten zeigten, wurden mehr als 5-mal häufiger mit erneuten Straftaten auffällig (Übermaß: OR $=5,34,95 \%$-KI [3,21, 8,87], $p<0,001$; für Missbrauch/Sucht: $\mathrm{OR}=5,48$, 95\%-KI $[2,68,11,19], p<0,001)$. An dieser Stelle soll angemerkt werden, dass Ritzels Kategorien wahrscheinlich eher subjektiv und möglicherweise nicht ganz trennscharf waren. Dennoch wurden seine Ergebnisse durch andere Untersuchungen gestützt. Auch Schmidt-Quernheim (2011) fand große Unterschiede zwischen den Rückfallraten von Patienten mit und ohne Substanzmissbrauch nach der Entlassung $\left(X^{2}=11,02, p=0,001, \omega=0,50\right)$. Diese fielen erwartungsgemäß zuungunsten konsumierender Patienten aus (OR=9,07, 95\%-KI [2,31, 35,65], $p=0,002)$. Ähnliche Werte berichtet auch Butz (2016). Konsumierende Teilnehmer hatten ein etwa 10-fach erhöhtes Risiko erneuter Straffälligkeit (OR=10,05, $95 \%$-KI [1,33, 75,73], $p$-Wert unbekannt).

Mittlere bis große Unterschiede in der erneuten Straffälligkeit werden auch für ehemalige §-64-Patienten berichtet, z.B. von Passow et al. $\left(2015 ; X^{2}=4,36, p=0,037, \omega=0,33\right)$ oder Pfaff $\left(1998 ; \mathrm{X}^{2}=13,65 \mathrm{a}, p<0,001 \mathrm{a}, \omega=0,58\right)$. Die Berechnung von ,odds ratios“ war in den meisten Fällen nicht möglich, da die Anzahl der Patienten, welche trotz Suchmittelrückfall straffrei blieben, zu gering war. Die deskriptiven Werte sprechen jedoch eine recht deutliche Sprache (z.B. Bezzel 2013; Dimmek et al. 2010).

\section{Nachsorge}

Die Untersuchung der Wirksamkeit forensischer Nachsorge weist einige methodische Probleme auf, da üblicherweise besonders Patienten mit ungünstigeren Ausgangsbedingungen und daraus folgend erhöhtem Rückfallrisiko der Nach- sorge zugewiesen wurden. Vor diesem Hintergrund sind die folgenden Ergebnisse zu interpretieren.

Die älteste Untersuchung zur Förderlichkeit einer ärztlichen Weiterbehandlung nach der Entlassung von \$-63Patienten stammte von Ritzel (1978). Die Daten zeigten einen deutlichen Unterschied $\left(X^{2}=20,45, p<0,001\right.$, $\omega=0,23$ ) zugunsten der Weiterbehandlung nach Entlassung $(\mathrm{OR}=0,38,95 \%$-KI $[0,25,0,58], p<0,001)$. Behandlungsergebnisse einer auf forensische Patienten spezialisierten Nachsorge berichtete Müller-Isberner (1996). Hinsichtlich erneuter schwerer Straftaten zeigte sich eine protektive Tendenz der forensischen Nachsorge $(\mathrm{OR}=0,45,95 \%$-KI $[0,15,1,36], p=0,158)$. Die Frage zur Qualität der Nachsorge in Abhängigkeit von der nachsorgenden Einrichtung untersuchten Seifert (2010) für allgemeine Rückfälligkeit und Seifert et al. (2018) für gewalttätige Deliktrückfälle. In beiden Studien konnten keine Unterschiede zwischen forensischen Kliniken, psychiatrischen Kliniken, niedergelassenen Psychiatern/Psychotherapeuten und sonstigen Professionen festgestellt werden. Eigene Berechnungen zeigten tendenziell mehr gewalttätige Deliktrückfälle bei durch forensische Kliniken nachbetreuten Patienten im Vergleich zu hausärztlich nachbetreuten Patienten $(\mathrm{OR}=2,00$, $95 \%$-KI $[0,88,4,55], p=0,095)$. Andererseits galt auch hier möglicherweise, dass vergleichsweise gefährliche Patienten eher von einer spezialisierten Klinik nachbehandelt wurden als vom Hausarzt. Zu Tätigkeitsschwerpunkten forensischer Ambulanzen lag eine Arbeit von Schmidt-Quernheim (2011) vor. Er fand keine Unterschiede zwischen der Behandlung von Patienten und Case Management durch die Ambulanz ( $\mathrm{OR}=1,96,95 \%$-KI $[0,48,7,99], p=0,348$ ). Hinsichtlich organisatorischer Belange erwies es sich als tendenziell günstiger, wenn es eine Instanz gab, welche die Nachsorge und daran beteiligte Akteure koordinierte, wenn für Krisenfälle ein allen bekannter Handlungsleitfaden vorlag und wenn die kontinuierliche Risikoeinschätzung nach festgelegten Kriterien verlief. Die genannten Tätigkeitsfelder sieht Schmidt-Quernheim (2011) als spezifische Kompetenz forensischer Ambulanzen. Eine weitere Aufgabe forensischer Nachsorge ist die möglichst frühe Intervention bei sich abzeichnenden Problemen. Über deren Einfluss berichtete Dimmek (2012). Für Patienten mit der Primärdiagnose einer Persönlichkeitsstörung (einschließlich sexuell abweichendem Verhalten) erwies sich dieses Vorgehen als sinnvoll $\left(\mathrm{X}^{2}=7,05, p=0,008, \omega=0,39\right)$. Interventionen nach dem ersten Verstoß folgte deutlich seltener ein Deliktrückfall als bei späterem Eingreifen $(\mathrm{OR}=0,18$, $95 \%$-KI $[0,05,0,07], p=0,010)$. Interventionen bei $\mathrm{Al}-$ koholkonsum bzw. Suchtverhalten (unabhängig von der Diagnose) schienen dagegen keinen Einfluss zu haben $\left(\mathrm{X}^{2}=0,03, p=0,853, \omega=0,03\right)$.

Über den Bewährungserfolg in Abhängigkeit von erfolgter Nachsorge für Patienten nach $\S 64$ StGB berich- 
teten Passow et al. (2015). Zwischen Patienten, welche mit und ohne forensischer Nachsorge entlassen wurden, zeigte sich kein statistisch bedeutsamer Unterschied $\left(\mathrm{X}^{2}=2,48\right.$, $p=0,115, \omega=0,17)$. Eine Tendenz sprach jedoch zugunsten ambulanter Nachsorge $(\mathrm{OR}=0,49,95 \%$-KI $[0,21,1,19]$, $p=0,117)$. Für eine Stichprobe alkoholkranker Patienten berichtete Pfaff (1998) tendenzielle Unterschiede zugunsten regelmäßiger Kontakte mit dem Bewährungshelfer, im Gegensatz zu keinen Kontakten $\left(\mathrm{X}^{2}=1,65, p=0,199, \omega=0,20\right)$.

\section{Psychologische und störungsassoziierte Variablen}

Unter diesem Abschnitt sollen all jene Faktoren zusammengefasst werden, die weniger die Außenwelt als das Innenleben entlassener Patienten berücksichtigten.

Für eine Patientenstichprobe, die nach $\S 63$ StGB untergebracht war, erfragte Ritzel (1978) die Hauptschwierigkeiten bei der Wiedereingliederung. Eine Reihe von Teilnehmern berichtete über Diskriminierung im sozialen und/oder im beruflichen Umfeld, z.B. durch geringere Entlohnung bei der Arbeit. Diese Diskriminierung wirkte sich negativ auf die Legalbewährung aus $(\mathrm{OR}=6,40,95 \%$-KI $[2,77$, 14,76], $p<0,001)$. Auch Belastungen durch ihre psychische Störung oder ein fortgeschrittenes Alter erschwerte einigen Teilnehmern die Wiedereingliederung (OR = 7,32, $95 \%$-KI $[3,05,17,55], p<0,001)$. Die statistisch bedeutsamste Auswirkung hatten allerdings Probleme im Umgang mit Behörden. Teilnehmer, welche eine negative Einstellung der Behörden beklagten, wurden mehr als 10-mal häufiger erneut straffällig als Teilnehmer, die Probleme bei der Wiedereingliederung verneinten $(\mathrm{OR}=10,36,95 \%$-KI $[3,77,28,40]$, $p<0,001)$. Leider ging Ritzel nicht näher darauf ein, worin diese negative Einstellung der Behörden bestand, sodass an dieser Stelle letztendlich nur spekuliert werden kann. Den Einfluss psychischer Stabilität erfasste Schmidt-Quernheim (2011). Es zeigten sich Unterschiede hinsichtlich der Legalbewährung in Abhängigkeit vom psychischen Zustand $\left(\mathrm{X}^{2}=8,05, p=0,005, \omega=0,30\right)$. Patienten, die durch ihre Behandler als eher nichtstabil eingeschätzt wurden, hatten etwa 6-mal häufiger neue Einträge im BZR als jene, die (weitgehend) stabil eingeschätzt wurden $(O R=6,10,95 \%$ KI [1,67, 22,19], $p=0,006)$. Auch Butz (2016) untersuchte die psychopathologische Entwicklung in seiner Stichprobe und erfragte Veränderungen in halbjährlichen $\mathrm{Ab}$ ständen von den Nachsorgeambulanzen. In seiner Untersuchung zeigte sich kein signifikanter Zusammenhang zwischen der psychopathologischen Entwicklung der letzten 6 Monate und der Deliktrückfälligkeit. Außerdem erfragte er die subjektive Einschätzung des Ambulanzpersonals hinsichtlich realistischer Zukunftspläne der Probanden. Auch hier unterschied sich der Bewährungserfolg nicht signifikant zwischen Patienten, bei denen (subjektiv) realistische
Zukunftspläne vorhanden waren, und jenen, bei denen diese Pläne nicht vorhanden waren.

Den Einfluss von Lebensvorstellungen und Erwartungen nach der Entlassung für ehemalige Patienten des Maßregelvollzugs nach $\S 64$ untersuchten auch Dimmek et al. (2010). Es zeigte sich, dass Patienten mit (subjektiv durch ihre Behandler eingeschätzten) eher realistischen und eher unrealistischen Vorstellungen etwa gleich häufig rückfällig wurden $\left(\mathrm{X}^{2}=0,36, p=0,551, \omega=0,05\right)$. In der Untersuchung von Butz (2016) unterschieden sich Patienten mit realistischen Plänen hinsichtlich ihrer Legalbewährung von jenen ohne derartige Pläne $\left(\mathrm{X}^{2}=16,22, p<0,01\right.$, $\omega=0,29)$. Es ergab sich ein $\mathrm{OR}=0,15$ zugunsten von Probanden mit realistischen Zukunftsplänen (95\%-KI [0,04, 0,61]). Der Einfluss des psychopathologischen Status der Probanden dagegen war nicht signifikant.

\section{Diskussion}

Die vorgestellten Studien unterliegen einigen methodischen Einschränkungen. Insgesamt sind die Rückfallquoten über alle Studien hinweg eher gering, verglichen mit ähnlichen Untersuchungen aus dem Regelvollzug (Jehle et al. 2016). Die Ergebnisse decken sich mit den Erkenntnissen internationaler Studien (Fazel et al. 2016). Auch wenn dieser Umstand allgemein erfreulich ist, ergeben sich hieraus für die statistische Auswertung einige Probleme. Die Gefahr, gerade kleine Effekte zu übersehen, ist insbesondere in Studien mit geringer Stichprobengröße besonders hoch. Des Weiteren erschweren die oft sehr unterschiedlichen Rückfalldefinitionen und eine große Variabilität der untersuchten Einflussfaktoren einen Vergleich der Ergebnisse.

Eine Einschränkung hinsichtlich der Repräsentativität betraf Untersuchungen an Teilnehmern, die nach § 64 StGB untergebracht waren. Die berichteten Untersuchungen wurden ausschließlich an Patienten durchgeführt, die ihre Therapie erfolgreich beendet hatten. Keine Studie untersuchte Delikt- oder Suchtmittelrückfälle bei Therapieabbrechern. Dies ist dem Umstand geschuldet, dass diese Subgruppe nach der Entlassung nur schwer erreichbar ist und kaum in ausreichender Zahl zu einer Studienteilnahme zu motivieren ist. Die vorliegenden Ergebnisse können daher nur für Patienten verallgemeinert werden, die ihre Behandlung nach $\S 64$ StGB erfolgreich beendeten. Weiterhin untersuchten die vorliegenden Studien ausschließlich unselektierte Stichproben. In diesen überwiegt der Anteil erwachsener Männer in der Regel sehr deutlich. Die vorliegenden Ergebnisse sind daher vor diesem Umstand zu interpretieren. Sie lassen keinerlei Aussagen für kleine, aber konstant vorhandene Subgruppen zu, allen voran Frauen, Jugendliche und Heranwachsende. Auch der Umstand, dass ein doch vergleichsweise großer Anteil der Maßre- 
gelpatienten einen anderen kulturellen Hintergrund als den deutschen hat, wird in keiner Studie berïcksichtigt. Auch für diese Patientengruppe sind die dargestellten Ergebnisse mit Vorsicht zu interpretieren. Weitere Einschränkungen ergeben sich aus der Auswertung von Studien mittels $\mathrm{X}^{2}$-Test. Aus diesem Test lassen sich zwar Unterschiede zwischen den Gruppen ableiten, aber keine Kausalzusammenhänge. Für einige Variablen stellt sich zusätzlich die Frage nach der Richtung des Zusammenhangs von Deliktrückfall und (vermeintlichem) Risikofaktor, welche durch den $\mathrm{X}^{2}$-Test unberücksichtigt bleiben.

In methodischer Hinsicht den Review betreffend müssen zwei Dinge einschränkend genannt werden. Zum einen erfolgte die Einschätzung des Verzerrungsrisikos aus Kapazitätsgründen nur durch die Autorin selbst. Auf die Einschätzung einer zweiten Person, wie es in gängigen ReviewLeitlinien empfohlen wird, musste verzichtet werden. Zum anderen erschwerte das spezielle Design der Untersuchungen, das stärker als in typischen klinischen Studien von äuBeren Umständen abhängt, die Suche nach angemessenen Bewertungskriterien.

Aus praktischer Sicht fällt weiterhin auf, dass sich internationale Studien häufiger auf die Qualität einer Einflussvariablen beziehen. Beispielsweise beschreiben Andrews und Bonta (2010) nicht nur das Vorhandensein einer Partnerschaft als Einflussfaktor, sondern betonen deren Qualität. Sie beschreiben z.B. die Art, wie die Partner miteinander umgehen, und die Verhaltenserwartungen aneinander als relevante Einflussgröße. Derart qualitative Merkmale werden in den vorliegenden Studien kaum adressiert. Ihre Kenntnis könnte möglicherweise einige der widersprüchlichen Ergebnisse aufklären und auch Hinweise für die Praxis liefern, worauf es sich zu achten lohnt. Um zu derartigen Ergebnissen zu kommen, ist es notwendig, über die rein auf Akteninformationen basierenden Studien hinauszugehen und Patienten direkt zu befragen, wie es einige Untersuchungen bereits tun. Nur so ist es möglich, die Qualität, z.B. von Beziehungen im Rahmen von Partnerschaft oder am Arbeitsplatz, zu erfassen. Die Ergebnisse von Hahn (2007) und Ritzel (1978) legen nahe, dass die Qualität dieser Beziehungen mindestens ebenso relevant ist wie ihr bloßes Vorhandensein.

Danksagung Unser Dank gilt Prof. Dr. Peter Sedlmeier für seine Unterstützung und hilfreichen Anmerkungen. Des Weiteren danken wir allen korrespondierenden Autoren, insbesondere Dr. Friedhelm Schmidt-Quernheim und Dr. Lutz Gretenkord, sowie den Reviewern für ihre hilfreichen Anmerkungen.

Funding Open Access funding provided by Projekt DEAL.

Interessenkonflikt P. Walde und V. Lungwitz geben an, dass kein Interessenkonflikt besteht.

Open Access Dieser Artikel wird unter der Creative Commons Namensnennung 4.0 International Lizenz veröffentlicht, welche die Nut- zung, Vervielfältigung, Bearbeitung, Verbreitung und Wiedergabe in jeglichem Medium und Format erlaubt, sofern Sie den/die ursprünglichen Autor(en) und die Quelle ordnungsgemäß nennen, einen Link zur Creative Commons Lizenz beifügen und angeben, ob Änderungen vorgenommen wurden.

Die in diesem Artikel enthaltenen Bilder und sonstiges Drittmaterial unterliegen ebenfalls der genannten Creative Commons Lizenz, sofern sich aus der Abbildungslegende nichts anderes ergibt. Sofern das betreffende Material nicht unter der genannten Creative Commons Lizenz steht und die betreffende Handlung nicht nach gesetzlichen Vorschriften erlaubt ist, ist für die oben aufgeführten Weiterverwendungen des Materials die Einwilligung des jeweiligen Rechteinhabers einzuholen.

Weitere Details zur Lizenz entnehmen Sie bitte der Lizenzinformation auf http://creativecommons.org/licenses/by/4.0/deed.de.

\section{Literatur}

Andrews DA, Bonta J (2010) The psychology of criminal conduct, 5. Aufl. Routledge, London

Andrews DA, Zinger I, Hoge RD, Bonta J, Gendreau P, Cullen FT (1990) Does correctional treatment work? A clinically relevant and psychologically informed meta-analysis. Criminology 28:369-404

Bezzel A (2013) Der Blick in den Spiegel ... - Standardisierte Erhebung der Ergebnisqualität im bayerischen Maßregelvollzug. Recht Psychiatr 31:123-128

Bischoff-Mews A (1998) Perspektiven des Maßregelvollzugs nach $\S 64$ StGB - Katamnestische Untersuchungen an alkoholkranken Straftätern im Maßregelvollzug. Med. Dissertation, FriedrichAlexander-Universität Erlangen-Nürnberg

Bonta J, Law M, Hanson K (1998) The prediction of criminal and violent recidivism among mentally disordered offenders: a meta-analysis. Psychol Bull 123:123-142. https://doi.org/10.1037/ 0033-2909.123.2.123

Butz M (2016) Forensisch-Psychiatrische Nachsorge in Bayern: Eine Untersuchung zu Deliktrückfällen und Prognosefaktoren bei bedingt entlassenen Maßregelvollzugspatienten ( $\S 63,64$ StGB). Med. Dissertation, Universität Regensburg

Cohen J (1988) Statistical power analysis for the behavioral sciences, 2. Aufl. Lawrence Erlbaum Associates, Hillsdale

Dahle K-P (2005) Psychologische Kriminalprognose. Wege zu einer integrativen Methodik für die Beurteilung der Rückfallwahrscheinlichkeit bei Strafgefangenen. Centaurus, Herbolzheim

Dimmek B (2012) Legalbewährung forensisch-psychiatrischer Patienten. Kriterien der Wiedereingliederung aus klinischer Sicht und die Wirksamkeit von Interventionen der Bewährungshilfe im Verlauf der Unterstellung unter Führungsaufsicht. Pabst, Lengerich

Dimmek B, Brunn D, Meier S, Stremmel M, Suer P, Westendarp A, Westendarp H (2010) Bewährungsverlauf und Wiedereingliederung suchtkranker Rechtsbrecher. Pabst, Lengerich

Dudeck M, Kopp D, Kuwert P, Drenkhahn K, Orlob S, Lütz HJ et al (2009) Die Prävalenz psychischer Erkrankungen bei Gefängnisinsassen mit Kurzzeitstrafe. Ergebnisse aus einer norddeutschen Haftanstalt. Psychiatr Prax 36(4):219-224

Fazel S, Fimińska Z, Cocks C, Coid J (2016) Patient outcomes following discharge from secure psychiatric hospitals: systematic review and meta-analysis. Br J Psychiatry 208(1):17-25

Gretenkord L (2001) Empirisch fundierte Prognosestellung im Maßregelvollzug nach $§ 63$ StGB (EFP-63), 2. Aufl. Deutscher Psychologen Verlag, Bonn

Hahn G (2007) Rückfallfreie Sexualstraftäter. Salutogenetische Faktoren bei ehemals im Maßregelvollzug behandelten Patienten. Psychiatrie-Verlag, Kassel 
Hartl C (2012) Wie erfolgreich ist die Behandlung im Maßregelvollzug nach $\S \S 63$ und 64 StGB? Eine Untersuchung anhand verschiedener Erfolgsmaße. Dissertation, Universität Regensburg

Jehle J-M, Albrecht H-J, Hohmann-Fricke S, Tetal C (2016) Legalbewährung nach strafrechtlichen Sanktionen. Forum-Verlag Godesberg, Mönchengladbach

Koch G (1988) Katamnesen bei suchtkranken Straftätern nach bedingter Entlassung aus dem Maßregelvollzug gem. § 64 StGB. Med. Dissertation, Medizinische Hochschule Hannover

Leygraf N (1988) Psychisch kranke Straftäter. Epidemiologie und aktuelle Praxis des psychiatrischen Maßregelvollzugs. Springer, Berlin

Moga C, Guo B, Schopflocher D, Harstall C (2012) Development of a quality appraisal tool for case series studies using a modified Delphi technique. Edmonton

Müller-Isberner JR (1996) Forensic psychiatric aftercare following hospital order treatment. Int J Law Psychiatry 19:81-86. https:// doi.org/10.1016/0160-2527(95)00023-2

Passow D, Prinz E, Maaß C, Wedler K, Bordel U, Schläfke D (2015) Legalbewährung und Konsumverhalten bei Probanden der forensischen Nachsorge nach Unterbringung in einer Entziehungsanstalt. Suchttherapie 17:90-95. https://doi.org/10.1055/s-0041107758

Pfaff H (1998) Ergebnisse einer prospektiven Katamnesestudie nach Entziehungstherapie gemäß 64 StGB bei Alkoholkranken. Nervenarzt 69:568-573. https://doi.org/10.1007/s001150050313
Ritzel G (1978) Unterbringung und Wiedereingliederung psychisch kranker Rechtsbrecher. Habilitation, Georg-August-Universität Göttingen

Schalast N (2019) Straffällige mit Suchtproblemen. Fakten, Erfahrungen und Ergebnisse der Essener Evaluationsstudie. Pabst, Lengerich

Schmidt-Quernheim F (2011) Evaluation der ambulanten Nachsorge forensischer Patienten ( $\$ 63 \mathrm{StGB})$ in Nordrhein-Westfalen. Dissertation, Universität Duisburg-Essen

von Schönfeld CE, Schneider F, Schröder T, Widmann B, Botthof U, Driessen M (2006) Prävalenz psychischer Störungen, Psychopathologie und Behandlungsbedarf bei weiblichen und männlichen Gefangenen. Nervenarzt 77(7):830-841

Seifert D (2010) Zur Gefährlichkeit ehemaliger Patienten des Maßregelvollzugs ( $\$ 63 \mathrm{StGB}$ ). Aktuelle Daten der Essener prospektiven Prognosestudie. Forens Psychiatr Psychol Kriminol 4:60-69

Seifert D, Klink M, Landwehr S (2018) Rückfalldaten behandelter Patienten im Maßregelvollzug nach $\S 63$ StGB. Forens Psychiatr Psychol Kriminol 12:136-148. https://doi.org/10.1007/s11757018-0471-6

Sterne JAC, Higgins JPT, Reeves BC (2014) A Cochrane risk of bias assessment tool: for non-randomized studies of interventions (ACROBAT-NRSI) 